\title{
Deswelling Induced Morphological Changes in Dual pH and Temperature Responsive Ultra- Low Crosslinked Poly ( $N$-isopropyl acrylamide)-co-Acrylic Acid Microgels
}

Molla R. Islam, Maddie Tumbarello and L. Andrew Lyon*

Department of Chemistry

Schmid College of Science and Technology, Chapman University, Orange, CA 92866

*Corresponding author: lyon@ chapman.edu

\begin{abstract}
Poly $(N$-isopropylacrylamide) microgels prepared without exogenous crosslinker are extremely "soft" as a result of their very low crosslinking density, with network connectivity arising only from the self-crosslinking of pNIPAm chains. As a result of this extreme softness, our group and others have taken interest in using these materials in a variety of bioengineering applications, while also pursuing studies of their fundamental properties. Here, we report deswelling triggered structural changes in poly ( $N$-isopropylacrylamide-co-acrylic acid) (ULC10AAc) microgels prepared by precipitation polymerization. Dynamic light scattering suggests that the deswelling of these particles not only depends on the collapse of the pNIPAm chains but is also influenced by the ionization state of the acrylic acid moieties present in the copolymer. The ULC10AAc microgel behaves like a traditional crosslinked pNIPAm microgel at $\mathrm{pH} 3.5$, showing a sharp decrease in the hydrodynamic diameter around the lower critical solution temperature (LCST) of pNIPAm. As the $\mathrm{pH}$ is increased to 4.5 we observe multiple transitions in the deswelling curve, suggesting inhomogeneity in the structure and/or composition of the microgels. At pH 6.5 the microgels cease
\end{abstract}


to be thermoresponsive over the studied temperature range due to increased charge repulsion between the fully deprotonated AAc groups and an increase in gel osmotic pressure due to solvated counterion ingress. Atomic force microscopy images of particles deposited at different temperatures reveal a temperature induced morphological change, with punctate structures forming inside microgels at $\mathrm{pH} 4.5$ and 6.5 and temperature above the gel volume phase transition temperature (VPTT).

\section{Introduction}

Microgels are colloidally stable cross-linked polymer networks with micron-scale dimensions. They are highly porous and soft in nature with this softness and porosity controllable by tailoring the crosslinking density of the network. ${ }^{1-5}$ Microgels can be made responsive to specific stimuli or analytes such as proteins, siRNA, DNA, temperature, $\mathrm{pH}$, ionic strength, magnetic fields, and/or electric fields by selecting specific co-monomers or through the addition of functional groups post-

polymerization. ${ }^{6-15}$ The ease with which microgel responsiveness, softness and porosity can be tailored makes them ideal candidates for a number of applications. 
The most widely studied thermo-responsive microgels are those composed of $\operatorname{poly}(N$ isopropylacrylamide) (pNIPAm), which are traditionally prepared by precipitation polymerization of $\mathrm{N}$-isopropylacrylamide (NIPAm) in presence of a cross-linking agent, such as $N, N^{\prime}-$ methylenebisacrylamide (BIS). ${ }^{2,3,16}$ Below $32{ }^{\circ} \mathrm{C}$, i.e., the volume phase transition temperature VPTT, the pNIPAm microgel adopts a swollen state, with the gel then collapsing upon increasing the temperature above the VPTT. The chemical and physical properties of pNIPAm microgels can be changed by controlling amount of BIS and by adding other functional comonomers during synthesis. ${ }^{6,9,17-22}$

The synthesis of microgels without adding any external crosslinker has been published recently. ${ }^{23}$, 24 The colloidal stability of polymer networks prepared without exogenous crosslinker has been explained as arising from the chain transfer reaction of propagating chains of pNIPAm resulting in a very low concentration of crosslinks/chain branches, which then form a loose network. Microgels prepared in this fashion are proposed to have very small core of higher density with an extended, loosely interconnected, polymer chain shell. Because of the low crosslinking density these microgel particles are extremely deformable. The Lyon and Brown groups have recently shown that highly deformable $\mathrm{pH}$ and thermoresponsive ULC microgels show promise as artificial platelets. ${ }^{25,26}$ When deposited on a solid substrate, ULC microgels show extreme deformability, with particles having a hydrodynamic diameter of $\sim 1.2 \mu \mathrm{m}$ adopting a dehydrated thickness of only a few nm when deposited on glass substrate. ${ }^{27,28}$ When deposited on an elastomeric substrate, these flattened microgels show a wrinkling pattern following substrate deformation, suggesting that the particles undergo plastic deformation when stressed in the dry state. ${ }^{28}$

We prepared self-crosslinked pNIPAm-co-AAc (ULC10AAc) particles and studied their deswelling response as a function of temperature and $\mathrm{pH}$. We used dynamic light scattering to 
calculate the diameter, phase analysis light scattering (PALS) to calculate the mobility of the particles in solution, and AFM to study the change in morphology induced by temperature. In general, we found that ULC10AAc microgels prepared in absence of cross-linking agent exhibit pNIPAm like deswelling characteristics at $\mathrm{pH}$ values below the $\mathrm{pKa}$ of AAc but have multiple volume phase transitions as a function of temperature at $\mathrm{pH}$ values above the pKa of AAc. We also noticed the evolution of punctate structures along the particle periphery with increasing temperature at a pH of 4.5. At $\mathrm{pH} 6.5$ we observed punctate structures homogenously distributed throughout the particle. We propose that the evolution of punctate structure is due to the stepwise collapse of the microgel particles with regions having higher local concentration of pNIPAm and the increased internal osmotic pressure associated with charged groups and hydrated counterion ingress.

\section{Experimental}

Materials. All reagents were purchased from Sigma-Aldrich unless noted otherwise. $\mathrm{N}$ isopropylacrylamide (NIPAm) was recrystallized from hexanes and vacuum dried prior to use. Acrylic acid (AAc), and ammonium persulfate (APS) were all used as received. Deionized water (DI) was obtained from a Thermo Scientific GenPure UV ×CAD system ( $\mathrm{GmbH}$, Germany) and was filtered to have a minimum resistivity of $18.2 \mathrm{M} \Omega \cdot \mathrm{cm}$. Dialysis tubing (Biotech CE, $1000 \mathrm{kD}$ MWCO, $31 \mathrm{~mm}$ Flat-width) was purchased from VWR. Acetic acid, formic acid and MES were purchased from VWR.

Poly(NIPAm-co-AAc) microgel Synthesis (ULC10AAc) 
Microgel particles containing acrylic acid as a co-monomer were prepared with a 90:10 ratio of co-monomers (NIPAm:AAc). Poly (N-isopropylacrylamide) (NIPAm) (1.49 g), and AAc (100 $\mu \mathrm{L})$ were added to $85 \mathrm{~mL}$ of $\mathrm{H}_{2} \mathrm{O}$ and stirred with a magnetic stir bar. Then the solution was filtered through a $0.2 \mu \mathrm{m}$ syringe filter into a three neck round bottom flask. An additional $10 \mathrm{~mL} \mathrm{H}_{2} \mathrm{O}$ was used to transfer and wash the beaker and filtered to the reaction vessel. A long waterless air condenser (CondenSyn Waterless condenser by Asynt) was placed in the center neck of the flask, and the solution was purged with $\mathrm{N}_{2}$ for $1 \mathrm{~h}$ while the temperature was equilibrated to $70{ }^{\circ} \mathrm{C}$ with constant stirring at $480 \mathrm{rpm}$. The reaction was initiated with APS solution (22.8 mg dissolved in 5 $\mathrm{mL}$ DI water, degassed and filtered with a $0.2 \mu \mathrm{m}$ syringe filter). The solution turned milky in less than $1 \mathrm{~min}$ and was allowed to proceed at $70^{\circ} \mathrm{C}$ for $6 \mathrm{~h}$. The reaction was stopped and cooled to room temperature before filtering the solution through glass wool to remove any coagulum.

\section{Purification of Microgel Particles}

Microgel particles were purified via dialysis (Biotech CE Dialysis Tubing, 1000 kDa MWCO, 31 mm Flat-width from VWR) against frequent changes of $\mathrm{DI}_{2} \mathrm{O}$ and by recording the conductivity until the conductivity of the dialysate matched the conductivity of the DI water.

\section{Microgel Deposition}

Microgels were deposited on functionalized $22 \mathrm{~mm}$ x $22 \mathrm{~mm}$ glass cover slips (VWR) according to procedures published elsewhere. ${ }^{27,29}$ First, glass slides were cleaned by sonication in different solvents for 30 minutes for each case: dilute Alconox solution, acetone, ethanol, and isopropanol. The cleaned glass surface was silanized with $1 \%(\mathrm{v} / \mathrm{v})$ APTMS in absolute ethanol on a table shaker for $2 \mathrm{~h}$ to render the surface cationic. The functionalized cover slips were stored in absolute ethanol until use. Before microgel deposition, the glass slides were rinsed with water and dried 
with $\mathrm{N}_{2}$. The cover slips were placed into different wells of a 24-well microwell plate with each well being filled with solutions of microgels. Microgels were centrifugally deposited onto the glass surface at $3700 \mathrm{rpm}(14.7 \mathrm{~g})$ for $10 \mathrm{~min}$ using an Eppendorf $5804 \mathrm{R}$ centrifuge equipped with a plate rotor at temperatures ranging from $20-40{ }^{\circ} \mathrm{C}$. We call this technique 'active deposition', which allows for the rapid formation of a homogeneous monolayer or sub-monolayer coverage of microgels on a substrate. ${ }^{30}$ After this step the cover slips were rinsed with DI water that had been equilibrated to the same temperature used for deposition and then dried with $\mathrm{N}_{2}$.

Microgels were also deposited on functionalized glass cover slips by passive deposition at $60{ }^{\circ} \mathrm{C}$. Passive deposition was done by incubating the microgel solution at $60{ }^{\circ} \mathrm{C}$ in an oven and dipping the glass slide in the solution for $30 \mathrm{~min}$. Afterwards the cover slips were gently rinsed with hot water to maintain the deposition temperature while removing excess microgels, and were dried with $\mathrm{N}_{2}$.

\section{Particle Characterization}

\section{Dynamic light scattering (DLS)}

DLS (Möbius, Wyatt technology) was used to determine the hydrodynamic radius $\left(R_{\mathrm{H}}\right)$ and electrophoretic mobility of the microgel particles was determined by phase analysis light scattering (PALS) at different temperatures in buffer solutions of $\mathrm{pH} 3.5,4.5$ and 6.5. The total ionic strength of the buffer solution was $10 \mathrm{mM}$ and additional $\mathrm{NaCl}$ was not added. Buffer solutions were filtered through a syringe filter prior to measurement (Supor membrane, $0.2 \mu \mathrm{m}$ pore width, PALL, Acrodisc). The solution of different microgel particles were transferred to a quartz cuvette and electrodes connected to a stopper was inserted into the solution. The whole set up was housed into a metal holder to place into the light scattering chamber. The scattering data was collected for 20 
s per acquisition with a total of 25 acquisitions. The measured intensity time correlation functions were analyzed using the cumulants method to calculate the diffusion coefficient. From this value, $R_{\mathrm{H}}$ was determined using the Stokes-Einstein equation. The data was analyzed by the software Dynamics 7 provided by Wyatt technology.

\section{Atomic Force Microscopy (AFM)}

The size and morphology of the microgels were further characterized by AFM imaging of glassdeposited microgel particles. Microgel monolayers were assembled on amine-functionalized glass cover slips in accordance with our previous work. ${ }^{28}$ An MFP-3D Origin AFM (Asylum Research, Santa Barbara, CA) was used to image microgel monolayers. Conical-shaped silicon AFM probes with $\mathrm{Al}$ reflex coating $\left(k=42 \mathrm{~N} \mathrm{~m}^{-1}\right)$ were mounted on the cantilever holder and operated in AC mode. AFM data was processed using the supplied software written in an IgorPro environment (Wavemetrics, Inc.).

\section{Optical Microscopy (OM)}

The morphology of the microgel in solution was characterized by Zeiss Axio Imager 2 microscope. The solution of microgels was dropped onto the microscope slides and covered with cover slips (thickness $1.5 \mathrm{~mm}$ ) before putting a drop of immersion oil (refractive index 1.515). Optical micrographs were recorded and processed with imaging software ZEN provided by Zeiss.

\section{Result and Discussion}

As described above, ULC10AAc microgels were dialyzed and lyophilized following synthesis. The lyophilized microgels were dissolved in different $\mathrm{pH}$ buffer solution of $10 \mathrm{mM}$ ionic strength without any added salt. Figure 1A shows photographs of ULC10AAc microgels in different $\mathrm{pH}$ 
solutions. We observe that the solution is semitransparent at $\mathrm{pH} 4.5$ where the AAc moieties are partially deprotonated, and the particles swelled due to electrostatic repulsion among the negatively charged AAc moieties and hydrated counterion ingress. At pH 6.5, the ULC10AAc solution becomes transparent confirming ionization of AAc and further swelling of the particles. OM micrographs (Figure $1 \mathrm{~B}-\mathrm{D}$ ) also confirm that the particles swell upon increasing the $\mathrm{pH}$. Note that the microgel particles become hard to visualize at $\mathrm{pH} 6.5$ because of the extreme swelling of the network. Again, this swelling is due to Coulombic repulsion between charged groups and hydrated counterion ingress. 

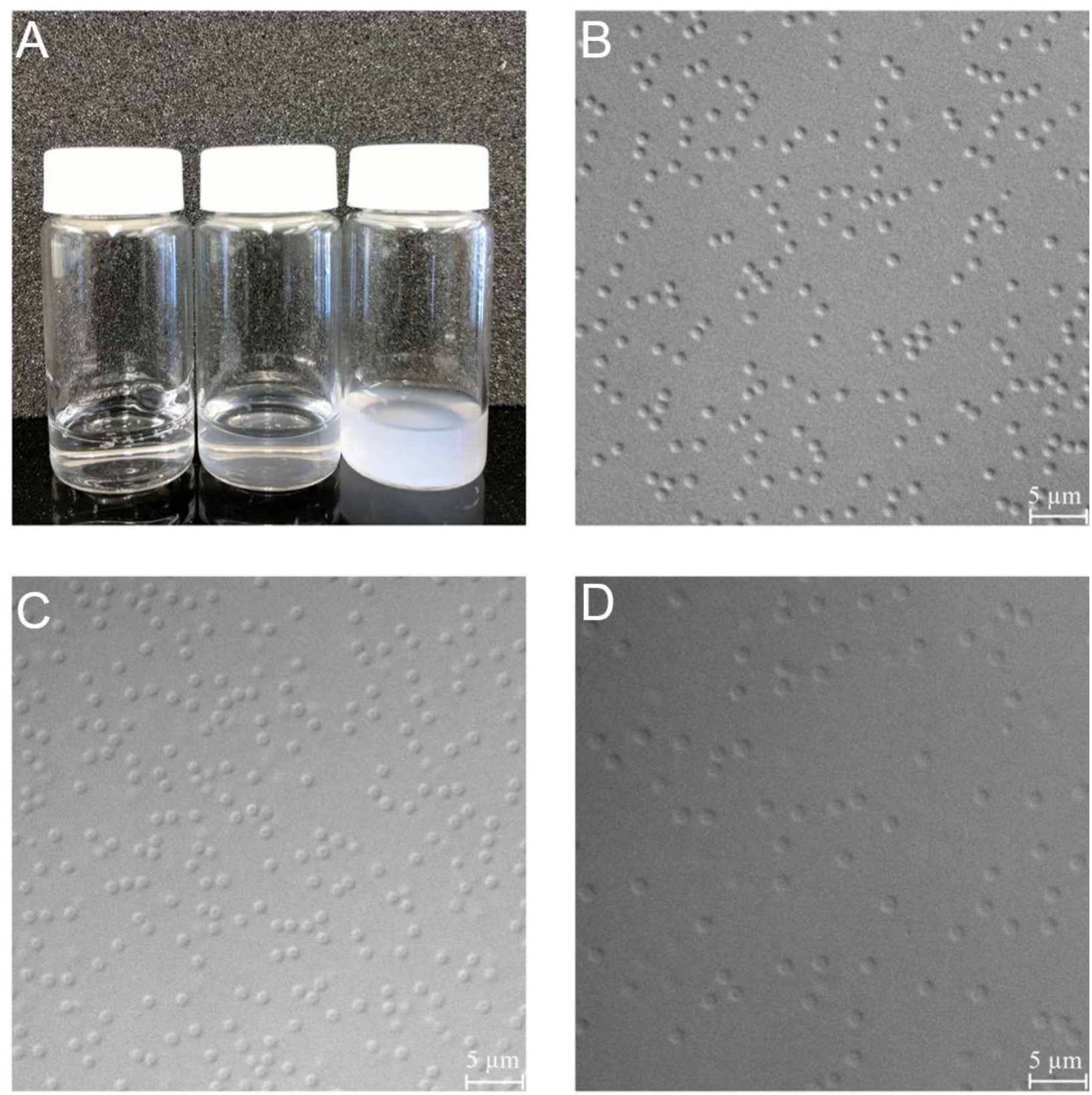

Figure 1. (A) Photograph of ultra-low crosslinked (ULC10AAc) microgels in different pH buffer solutions (left to right pH 6.5, 4.5 and 3.5 respectively); (B-D) Optical micrographs of ULC10AAc microgels in different $\mathrm{pH}$ solutions. Here B represents the microgels in $\mathrm{pH} 3.5$ while $\mathrm{C}$ and $\mathrm{D}$ represent the microgels in $\mathrm{pH} 4.5$ and 6.5 respectively. 
Figure 2A shows the deswelling curve of the ULC10AAc at $\mathrm{pH}$ 3.5. The observed deswelling curve is very similar to those reported for BIS crosslinked pNIPAm microgels, which is not surprising considering that the degree of crosslinking has only a small impact on the shape of the deswelling curve of pNIPAm microgels. ${ }^{16,31-33}$ Based on light scattering data, it has been proposed previously that crosslinked microgel particles undergo a three stage collapse. ${ }^{16,32}$ Initially, the core of the particle collapses and then the shell undergoes the subsequent shrinkage followed by final core collapse. We investigated the electrophoretic mobility of the particles at different temperature at $\mathrm{pH} 3.5$ to reveal any unobserved transition due to different ionic states of the microgels that were not observed by monitoring the diameter change due to thermoresponsivity of the microgels. The temperature associated transition in electrophoretic mobility is known as Electrokinetic Transition Temperature (ETT). The microgel ETT is related to the charge distribution and overall change density along the periphery of the microgels. We observed a very small increase in mobility at temperature below $\sim 31{ }^{\circ} \mathrm{C}$ suggesting little or no change in charge distribution or ionic state in the periphery of the microgels, despite the dramatic shrinkage of the microgel over that same temperature range. However, the mobility increases dramatically above $31{ }^{\circ} \mathrm{C}$ until $\sim 45^{\circ} \mathrm{C}$ while a more modest change in diameter is observed over that temperature range. This large increase in mobility indicates the overall increase in peripheral charge density with temperature due to the change in internal microgel structure. We would like to highlight here that the largest changes in diameter and mobility occur at temperatures that differ by $\sim 3.5^{\circ} \mathrm{C}$. Similar observations have been reported for cross-linked pNIPAm-AAc microgels and were associated with the nature of particle collapse and the charge distribution along the particle periphery during the different, spatiallylocalized, stages of collapse. ${ }^{16,32}$ 
Figure 2B shows the deswelling curve of ULC10AAc microgels at $\mathrm{pH}$ 4.5. The AAc moieties of the microgel should be partially deprotonated since to the $\mathrm{pK}_{\mathrm{a}}$ of AAc is $\sim 4.25$. We observed the general trend of a large decrease in particle diameter with increase in temperature until $\sim 31{ }^{\circ} \mathrm{C}$ with a smaller increase in diameter to $45{ }^{\circ} \mathrm{C}$ and then no change in diameter as we increase the temperature to $60{ }^{\circ} \mathrm{C}$. To reveal any intermediate transition, we recorded the deswelling of microgels at a very slow increase in temperature by DLS. A high-resolution scan of the DLS data (Figure 2C) suggests that the change in diameter goes through multiple transitions, which were not observed for the ULC10AAc microgel at $\mathrm{pH}$ 3.5. This observation has not been reported elsewhere for a self-crosslinked microgel system. Similar observations have been reported previously for a crosslinked pNIPAm microgel system in presence of sodium dodecyl sulfate (SDS) which was explained by the inhomogeneity of the chain length between two neighboring crosslinks. ${ }^{34}$ In this case, the transitions are separated by $\sim 5{ }^{\circ} \mathrm{C}$ and are presumably associated with different environments in the microgels that are rich in one of the two co-monomers. The partial deprotonation of AAc moieties of ULC10AAc at $\mathrm{pH} 4.5$ may influence the transition by frustrating the collapse of the pNIPAm chain due to Coulombic repulsion in AAc-rich regions. We also notice a smaller change in electrokinetic mobility of the particles with temperature up to $34{ }^{\circ} \mathrm{C}$. When we compare the temperatures associated with the most significant changes in diameter and mobility of the particles a temperature difference between those transitions of $\sim 7^{\circ} \mathrm{C}$ is observed.

Figure 2D shows the temperature dependent deswelling response of the ULC10AAc particles at $\mathrm{pH}$ 6.5. It should be noted here that the DLS data shown are likely associated with high degree of error; obtaining accurate DLS data for these particles is challenging due to the very low refractive index contrast between the particles and the surrounding medium (water). Despite this difficulty, it is clear that ULC10AAc particles lose their thermoresponsivity at $\mathrm{pH} 6.5$ due to the large 
increase in internal osmotic pressure associated with Coulombic repulsion and hydrated counterion ingress. Figure 2E shows the relative change in diameter of the ULC10AAc particles as a function of temperature at different $\mathrm{pH}$. 



Figure 2. Swelling plots of ULC10AAc microgels as a function of temperature at (A) $\mathrm{pH} 3.5$; (B) $\mathrm{pH} 4.5$; (C) high resolution scan at $\mathrm{pH} 4.5$ and (D) $\mathrm{pH}$ 6.5. Each data point in $\mathrm{A}, \mathrm{B}, \mathrm{C}$ and $\mathrm{D}$ 
represents the average of at least three independent measurements and the error bars are calculated as the standard deviation for those values. Here, (E) represents a comparison plot of the change in diameter of microgel at different $\mathrm{pH}$ as a function of temperature. Here the symbols $\triangle, \square$ and $\square$ represent microgels in $\mathrm{pH} 3.5,4.5$ and 6.5 respectively.

To better understand whether any heterogeneous structural changes occur during the deswelling of the microgels, we investigated the morphology of the microgel particles deposited on a solid substrate at different temperatures by atomic force microscopy (AFM). Figure 3A shows microgels deposited by active deposition at different temperatures at $\mathrm{pH}$ 3.5. We can see how the diameter of the microgel particles dramatically reduces from $1.2 \mu \mathrm{m}$ at $20^{\circ} \mathrm{C}$ to $350 \mathrm{~nm}$ at $40{ }^{\circ} \mathrm{C}$ (Figure 3A-C) without any significant change in the height of the particles. In a previous report, the temperature induced transition below $35^{\circ} \mathrm{C}$ was proposed as a core-localized transition whereas transition in between $<35^{\circ} \mathrm{C}$ to $38^{\circ} \mathrm{C}$ was attributed to a shell-localized transition for crosslinked pNIPAm microgel. ${ }^{16}$ Here, we do not observe any well-defined morphological transition that can be as attributed to core or shell collapse. Instead, we notice an overall change in diameter triggered by the deswelling of the pNIPAm network. Figure 3D shows microgel particles deposited by passive deposition where positively functionalized cover slips were dipped in a microgel solution incubated at $60{ }^{\circ} \mathrm{C}$ and kept for 30 minutes. It is evident from the AFM images that the particles become smaller and the height of the particles increases significantly. Figure 3E represents how the diameter and height of the microgel particles changes with temperature. It is to be noted here that the microgel particles deposited at $60{ }^{\circ} \mathrm{C}$ were deposited by passive deposition, whereas active deposition was used at all other temperatures. 

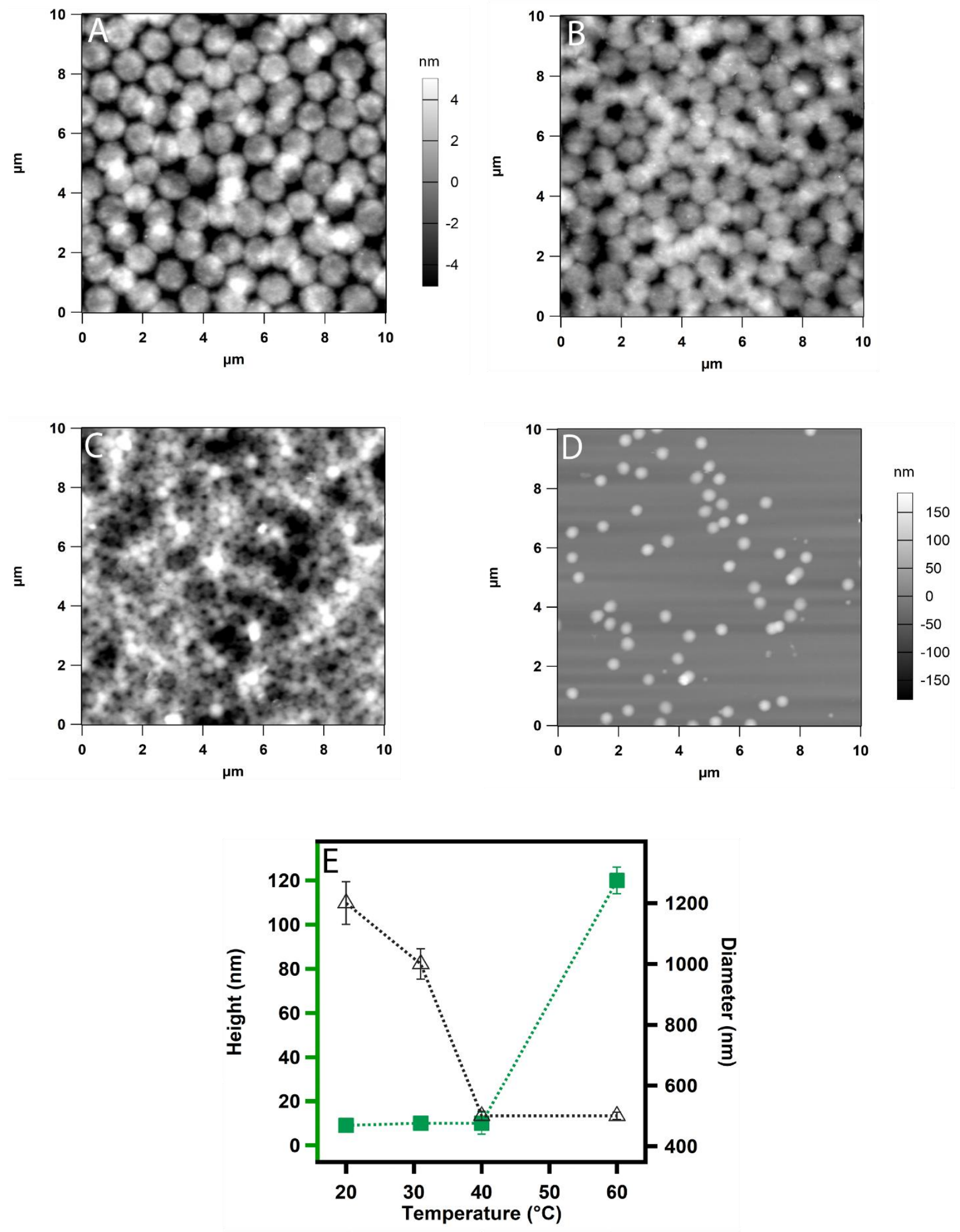
Figure 3. AFM images (A-C) of actively deposited ULC10AAc microgels from pH 3.5 solution. The deposition was carried out at (A) $20{ }^{\circ} \mathrm{C}$ (B) $32{ }^{\circ} \mathrm{C}$ and (C) $40{ }^{\circ} \mathrm{C}$. Passive deposition was used for sample (D) at $60{ }^{\circ} \mathrm{C}$. The z-scale shown with panel A is same for B and C. Here (E) shows the plot of the change in height and diameter of the particles as a function of temperature. Each data point in E represents the average of at least three independent measurements and the error bars are calculated as the standard deviation for those values.

We observe a very different structural change with the temperature when the particles are deposited from $\mathrm{pH} 4.5$ solution. Figure 4A shows the AFM image of microgel particles deposited at $20^{\circ} \mathrm{C}$ where they were in swollen state. As we increase the temperature to $31{ }^{\circ} \mathrm{C}$ and $33{ }^{\circ} \mathrm{C}$, we notice the appearance of punctate structure along the periphery of the microgels with a slight decrease in diameter as shown in Figure 4B and 4C. When the temperature is $35^{\circ} \mathrm{C}$ (Figure 4D) the punctate structure spreads homogeneously throughout the whole microgel. Whereas this specific structural change has not been reported previously in ULC microgels, previous reports have predicted mass segregation inside the particles for different crosslinked systems. ${ }^{33,}{ }^{35}$ Increasing the temperature to $40{ }^{\circ} \mathrm{C}$ results in particle size decreases with absence of punctate structure (Figure $4 \mathrm{E}$ ). At $60{ }^{\circ} \mathrm{C}$ when the microgels were deposited by passive deposition, the particles get even smaller as shown in Figure 4F. As we plot the change in diameter and height of the particles as a function of temperature we notice a significant change in diameter and height with temperature. 

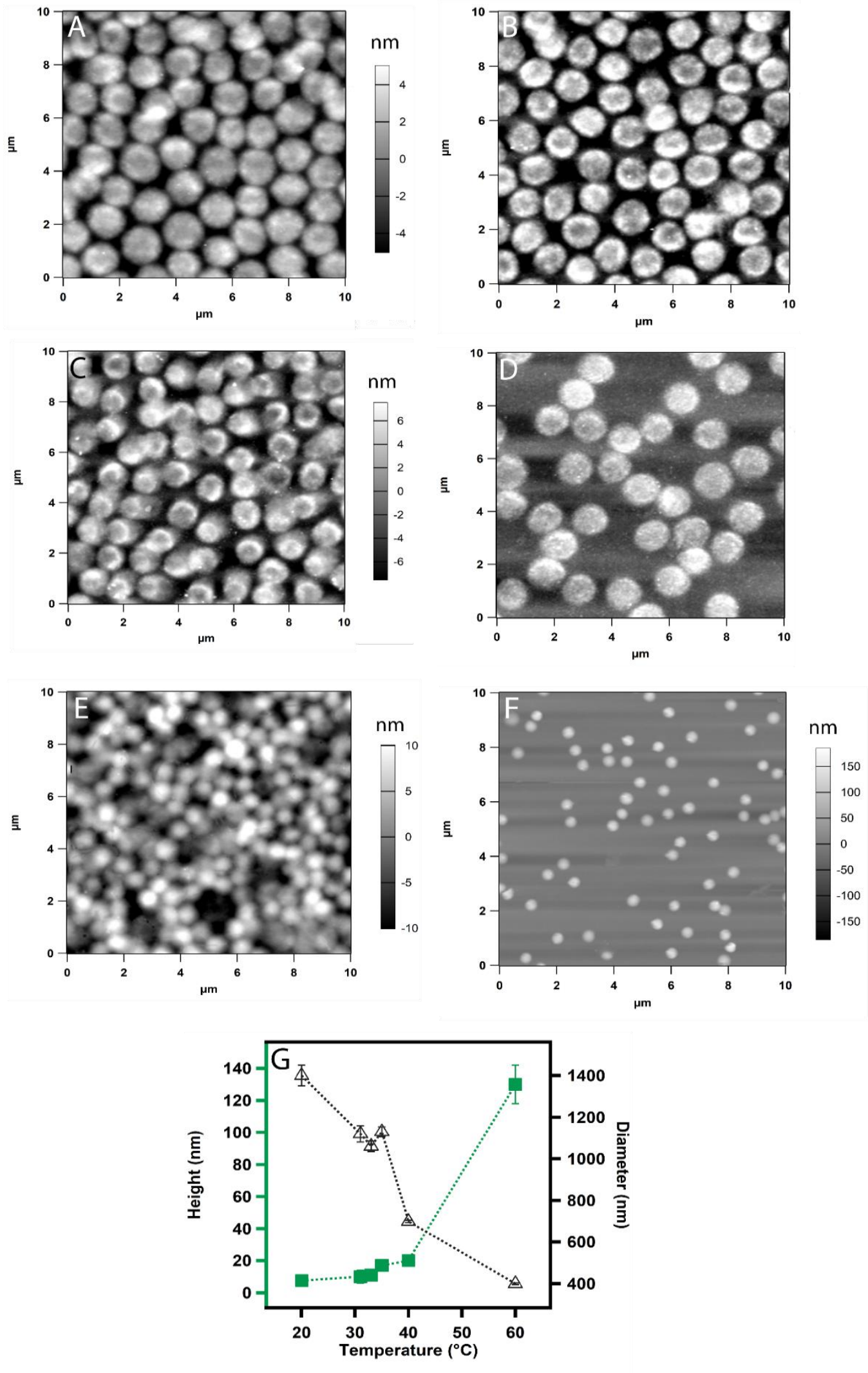
Figure 4. AFM images (A-E) of actively deposited ULC10AAc microgels from $\mathrm{pH} 4.5$ solution. The deposition was carried out at (A) $20^{\circ} \mathrm{C}$ (B) $31{ }^{\circ} \mathrm{C}$ (C) $33{ }^{\circ} \mathrm{C}$ (D) $35^{\circ} \mathrm{C}$ and (E) $40{ }^{\circ} \mathrm{C}$. Passive deposition was used for sample $(\mathrm{F})$ at $60{ }^{\circ} \mathrm{C}$. The z-scale shown with panel $\mathrm{A}$ is same for $\mathrm{B}$ and the z-scale shown with panel C is same for D. Here $(G)$ represents the plot of the change in height and diameter of the particles at different temperatures. Each data point represents the average of at least three independent measurements and the error bars are calculated as the standard deviation for those values.

Figure 5 shows the structural change in the microgels as a function of temperature when they are deposited from a pH 6.5 solution. At this $\mathrm{pH}$ the AAc moieties are nearly 100\% deprotonated and microgel network collapse should be frustrated by the electrostatic repulsion and counterionassociated increases in osmotic pressure. Despite this expectation, we still observe a punctate structure at the particles periphery at $40{ }^{\circ} \mathrm{C}$ (Figure 5B) whereas punctate structures were evenly developed at $60^{\circ} \mathrm{C}$ (Figure 5C). We also notice a relatively small decrease in diameter and height. Figure 5D shows a small increase in the height and small decrease in the diameter of the particles with temperature. 

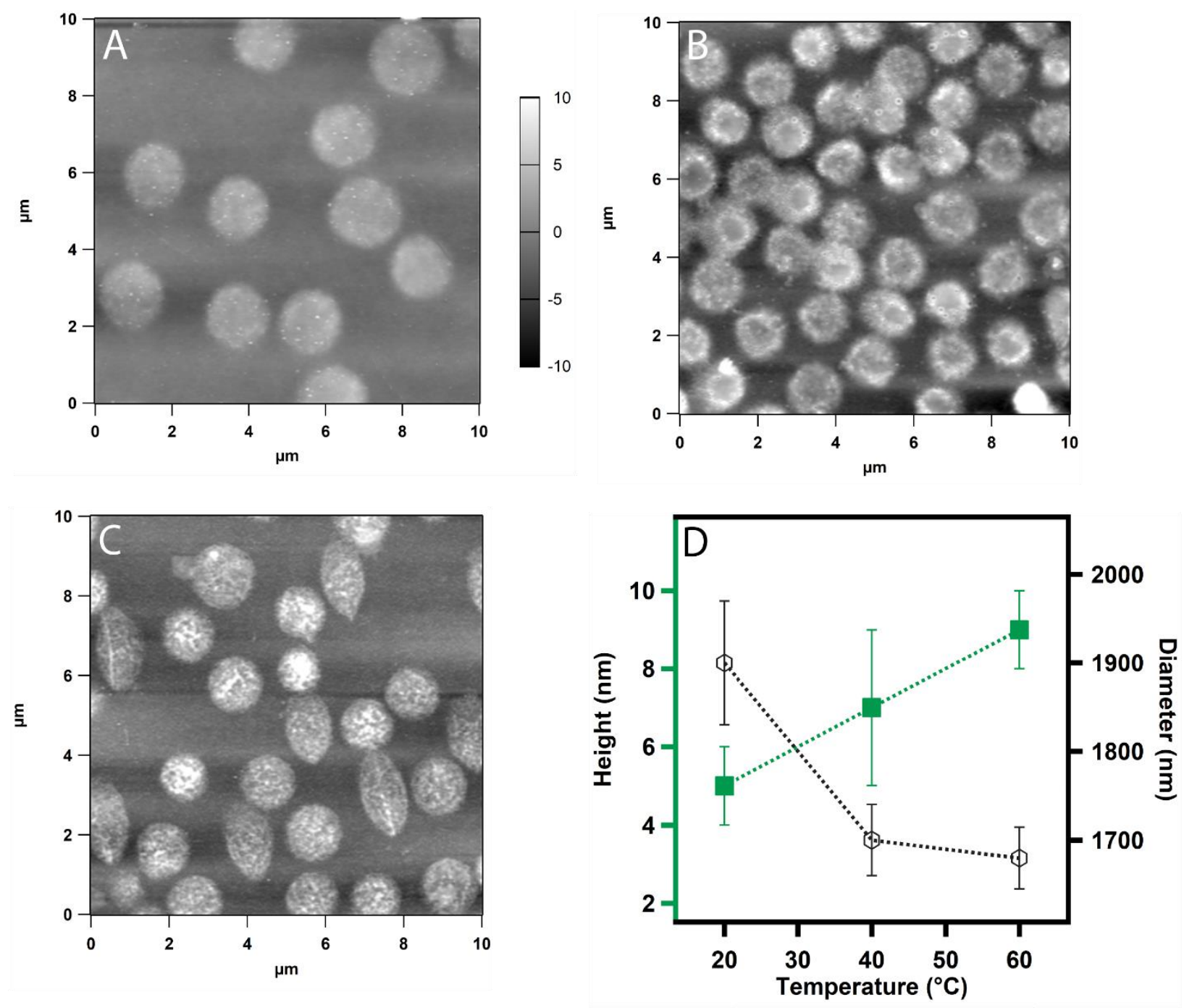

Figure 5. AFM Images (A and B) of actively deposited ULC10AAc microgels from pH 6.5 solution. The deposition was carried out at $20^{\circ} \mathrm{C}(\mathrm{A})$ and $40^{\circ} \mathrm{C}(\mathrm{B})$. Passive deposition was used for sample (C) at $60{ }^{\circ} \mathrm{C}$. The Z-scale shown with panel $\mathrm{A}$ is same for both $\mathrm{B}$ and $\mathrm{C}$. The temperatures were selected based on the deswelling plot of the microgel. Here (D) represents the plot of the change in height and diameter of the particles at some specific temperatures. Each data 
point in D represents the average of at least three independent measurements and the error bars are calculated as the standard deviation for those values.

The data presented above reveal some interesting subtleties in the temperature-induced collapse of dual temperature/pH responsive microgels. When observed on the macroscopic and single particle scale, the microgel behavior seems simple and straightforward (e.g. photographs shown in Figure 1); increases in $\mathrm{pH}$ result in greater microgel swelling due to deprotonation of the AAc groups.

However, when multiple different light scattering methods are used to observe particle collapse, significantly more complex behavior is revealed. Taken together, the data presented in Figures 25 paint a picture of structural and compositional heterogeneity within the microgels. Differences between the VPTT determined by DLS and the ETT determined by PALS strongly suggest that surface enrichment of charged groups happens after the main particle volume transition. This is likely due to the large volume change being associated with pNIPAm-rich segments, while the more AAc rich segments collapse at a higher temperature, at which point the microgel surface charge undergoes a large change. This multi-transition behavior is clearly observed in Figure 2B,C under conditions of partial AAc deprotonation.

Likewise, AFM data suggest that the temperature induced deswelling creates small domains due to the collapse of the pNIPAm chain in the pNIPAm-rich area. This domain formation is only evident in the $\mathrm{pH}$ above the $\mathrm{pKa}$ of AAc confirming the less random nature of the polymer chains and the effect of the columbic repulsion forming the microgels. These domains are formed $\sim 31$ ${ }^{\circ} \mathrm{C}$ at the periphery first and then spread to the whole microgel with increase in temperature. The 
multiple transition observed from the deswelling study at $\mathrm{pH} 4.5$ and the punctate domain formation above $31{ }^{\circ} \mathrm{C}$ may be related to the odd phase segregation from the opposite thermoresponsive nature of uncharged pNIPAm-rich and charged AAc rich regions of the microgels. This observation enrich our existing understanding of the temperature induced structural change inside the microgel network.

\section{Conclusions}

We have shown that dual temperature/pH responsive ULC microgel particles display complex deswelling and phase segregation behavior. This is likely due to the inhomogeneous distribution of the NIPAm and AAc co-monomers, creating pNIPAm and AAc rich domains within the network. This heterogeneity is revealed through the difference between the temperature of VPTT and ETT, which increases significantly as the ULC particles transition from an uncharged state to charged state. The evolution of a punctate internal structure at $\mathrm{pH}$ above 4.5 also confirms polymer segregated due to inhomogeneous co-monomer distribution. As the temperature increase initiates the collapse of the pNIPAm rich segments, AAc deprotonation frustrates chain collapse by increasing the osmotic pressure inside the microgels. Given the increasing importance of these unique particles in biotechnology applications, we anticipate that the deeper understanding of ULC particle structure obtained here will be important as more sophisticated ULC particle designs emerge in more advanced applications.

\section{References}

1. Kawaguchi, H. Functional polymer microspheres. Prog. Polym. Sci. 2000, 25 (8), 11711210 DOI: 10.1016/S0079-6700(00)00024-1. 
2. Pelton, R. Temperature-sensitive aqueous microgels. Adv. Colloid Interface Sci. 2000, 85 (1), 1-33 DOI: 10.1016/S0001-8686(99)00023-8.

3. Pelton, R. H.; Chibante, P. Preparation of aqueous latexes with $\mathrm{N}$-isopropylacrylamide. Colloids Surf. 1986, 20 (3), 247-56 DOI: 10.1016/0166-6622(86)80274-8.

4. Pichot, C.; Taniguchi, T.; Delair, T.; Elaissari, A. Functionalized thermosensitive latex particles: Useful tools for diagnostics. J. Dispersion Sci. Technol. 2003, 24 (3 \& 4), 423-437 DOI: 10.1081/DIS-120021799.

5. Zhang, H.; Mardyani, S.; Chan, W. C. W.; Kumacheva, E. Design of Biocompatible Chitosan Microgels for Targeted pH-Mediated Intracellular Release of Cancer Therapeutics. Biomacromolecules 2006, 7 (5), 1568-1572 DOI: 10.1021/bm050912z.

6. Hoare, T.; Pelton, R. Titrametric characterization of $\mathrm{pH}$-induced phase transitions in functionalized microgels. Langmuir 2006, 22 (17), 7342-7350 DOI: 10.1021/la0608718.

7. Hoare, T.; Pelton, R. Engineering glucose swelling responses in poly $(\mathrm{N}-$ isopropylacrylamide)-based microgels. Macromolecules 2007, 40 (3), 670-678 DOI: 10.1021/ma062254w.

8. Campbell, S. B.; Hoare, T. Externally addressable hydrogel nanocomposites for biomedical applications.Curr. Opin. Chem. Eng. 2014, 4, 1-10 DOI: 10.1016/j.coche.2013.12.003. 9. Hoare, T.; Pelton, R. Highly $\mathrm{pH}$ and temperature responsive microgels functionalized with vinylacetic acid. Macromolecules 2004, 37 (7), 2544-2550 DOI: 10.1021/ma035658m.

10. Smeets, N. M. B.; Hoare, T. Designing responsive microgels for drug delivery applications. J. Polym. Sci. A 2013, 51 (14), 3027-3043 DOI: 10.1002/pola.26707. 
11. Nayak, S.; Lee, H.; Chmielewski, J.; Lyon, L. A. Folate-mediated cell targeting and cytotoxicity using thermoresponsive microgels. J. Am. Chem. Soc. 2004, 126 (33), 10258-10259 DOI: $10.1021 / \mathrm{ja} 0474143$.

12. Kim, J.; Singh, N.; Lyon, L. A. Label-free biosensing with hydrogel microlenses. Angew. Chem., Int. Ed. 2006, 45 (9), 1446-1449 DOI: 10.1002/anie.200503102.

13. Nolan, C. M.; Serpe, M. J.; Lyon, L. A. Thermally modulated insulin release from microgel thin films. Biomacromolecules 2004, 5 (5), 1940-1946 DOI: 10.1021/bm049750h.

14. Serpe, M. J.; Yarmey, K. A.; Nolan, C. M.; Lyon, L. A. Doxorubicin Uptake and Release from Microgel Thin Films. Biomacromolecules 2005, 6 (1), 408-413 DOI: 10.1021/bm049455x.

15. Smith, M. H.; Lyon, L. A. Multifunctional Nanogels for siRNA Delivery. Acc. Chem. Res. 2012, 45 (7), 985-993 DOI: 10.1021/ar200216f.

16. Pelton, R. H.; Pelton, H. M.; Morphesis, A.; Rowell, R. L. Particle sizes and electrophoretic mobilities of poly(N-isopropylacrylamide) latex. Langmuir 1989, 5 (3), 816-18 DOI: 10.1021/la00087a040.

17. O'Reilly, R. K.; Joralemon, M. J.; Hawker, C. J.; Wooley, K. L. Facile syntheses of surfacefunctionalized micelles and shell cross-linked nanoparticles. J. Polym. Sci. A2006, 44 (17), 52035217 DOI: 10.1002/pola.21602.

18. Slater, M.; Snauko, M.; Svec, F.; Frechet, J. M. J. "Click Chemistry" in the Preparation of Porous Polymer-Based Particulate Stationary Phases for $\mu$-HPLC Separation of Peptides and Proteins. Anal. Chem. 2006, 78 (14), 4969-4975 DOI: 10.1021/ac060006s.

19. Vinogradov, S. V.; Bronich, T. K.; Kabanov, A. V. Nanosized cationic hydrogels for drug delivery: preparation, properties and interactions with cells. Adv. Drug Delivery Rev. 2002, 54 (1), 135-147 DOI: 10.1016/S0169-409X(01)00245-9. 
20. Saunders, B. R.; Crowther, H. M.; Vincent, B. Poly[(methyl methacrylate)-co-(methacrylic acid)] Microgel Particles: Swelling Control Using pH, Cononsolvency, and Osmotic Deswelling. Macromolecules 1997, 30 (3), 482-487 DOI: 10.1021/MA961277F.

21. Islam, M. R.; Serpe, M. J. Penetration of Polyelectrolytes into Charged Poly(Nisopropylacrylamide) Microgel Layers Confined between Two Surfaces. Macromolecules 2013, 46 (4), 1599-1606 DOI: 10.1021/ma302637n.

22. Hoare, T.; Pelton, R. Functional group distributions in carboxylic acid containing poly(Nisopropylacrylamide) microgels. Langmuir 2004, 20 (6), 2123-2133 DOI: 10.1021/la0351562.

23. Gao, J.; Frisken, B. J. Cross-Linker-Free N-Isopropylacrylamide Gel Nanospheres. Langmuir 2003, 19 (13), 5212-5216 DOI: 10.1021/la0269762.

24. Gao, J.; Frisken, B. J. Influence of Reaction Conditions on the Synthesis of Self-CrossLinked N-Isopropylacrylamide Microgels. Langmuir 2003, 19 (13), 5217-5222 DOI: 10.1021/la034207s.

25. Brown, A. C.; Stabenfeldt, S. E.; Ahn, B.; Hannan, R. T.; Dhada, K. S.; Herman, E. S.; Stefanelli, V.; Guzzetta, N.; Alexeev, A.; Lam, W. A.; Lyon, L. A.; Barker, T. H. Ultrasoft microgels displaying emergent platelet-like behaviours. Nat. Mater. 2014, 13 (12), 1108-1114 DOI: $10.1038 /$ nmat4066.

26. Sproul, E. P.; Nandi, S.; Roosa, C.; Schreck, L.; Brown, A. C. Biomimetic Microgels with Controllable Deformability Improve Healing Outcomes. Advanced Biosystems 0 (0), 1800042 DOI: doi:10.1002/adbi.201800042.

27. Bachman, H.; Brown, A. C.; Clarke, K. C.; Dhada, K. S.; Douglas, A.; Hansen, C. E.; Herman, E.; Hyatt, J. S.; Kodlekere, P.; Meng, Z.; Saxena, S.; Spears Jr, M. W.; Welsch, N.; Lyon, 
L. A. Ultrasoft, highly deformable microgels. Soft Matter 2015, 11 (10), 2018-2028 DOI: 10.1039/C5SM00047E.

28. Gaulding, J. C.; Spears, M. W.; Lyon, L. A. Plastic deformation, wrinkling, and recovery in microgel multilayers. Polym. chem. 2013, 4 (18), 4890-4896 DOI: 10.1039/C3PY00173C.

29. Welsch, N.; Lyon, L. A. Oligo(ethylene glycol)-sidechain microgels prepared in absence of cross-linking agent: polymerization, characterization and variation of particle deformability. PLoS One 2017, 12 (7), e0181369/1-e0181369/23 DOI: 10.1371/journal.pone.0181369.

30. South, A. B.; Whitmire, R. E.; García, A. J.; Lyon, L. A. Centrifugal Deposition of Microgels for the Rapid Assembly of Nonfouling Thin Films. ACS Appl. Mater. Interfaces 2009, 1 (12), 2747-2754 DOI: 10.1021/am9005435.

31. Daly, E.; Saunders, B. R. Temperature-dependent electrophoretic mobility and hydrodynamic radius measurements of poly( $\mathrm{N}$-isopropylacrylamide) microgel particles: structural insights. Phys. Chem. Chem. Phys. 2000, 2 (14), 3187-3193 DOI: 10.1039/b002678f.

32. Daly, E.; Saunders, B. R. Temperature-dependent electrophoretic mobility and hydrodynamic radius measurements of poly( $\mathrm{N}$-isopropylacrylamide) microgel particles: structural insights. Phys. Chem. Chem. Phys. 2000, 2 (14), 3187-3193 DOI: 10.1039/b002678f.

33. Hoare, T.; Pelton, R. Electrophoresis of functionalized microgels: morphological insights. Polymer 2005, 46 (4), 1139-1150 DOI: 10.1016/j.polymer.2004.11.055.

34. Chi, W.; Shuiqin, Z.; F., A.-y. S. C.; Suhong, J. Volume phase transition of spherical microgel particles. Macromol. Chem. Phys. 1996, 240 (1), 123-136 DOI: doi:10.1002/apmc.1996.052400111.

35. Saunders, B. R. On the Structure of Poly(N-isopropylacrylamide) Microgel Particles. Langmuir 2004, 20 (10), 3925-3932 DOI: 10.1021/la036390v. 
